OPEN ACCESS

Edited by:

Takeshi lgawa

Hiroshima University, Japan

Reviewed by:

Frederic Guy Brunet,

UMR5242 Institut de Génomique Fonctionnelle de Lyon (IGFL), France

Rita Rebollo,

Institut National de la Recherche Agronomique (INRA), France

*Correspondence:

Michihiko Ito

ito@sci.kitasato-u.ac.jp

Specialty section:

This article was submitted to

Evolutionary and Population Genetics,

a section of the journal

Frontiers in Genetics

Received: 29 August 2021 Accepted: 13 January 2022

Published: 31 January 2022

Citation:

Suda K, Hayashi SR, Tamura K,

Takamatsu $N$ and Ito $M$ (2022) Activation of DNA Transposons and Evolution of piRNA Genes Through

Interspecific Hybridization in

Xenopus Frogs.

Front. Genet. 13:766424.

doi: 10.3389/fgene.2022.766424

\section{Activation of DNA Transposons and Evolution of piRNA Genes Through Interspecific Hybridization in Xenopus Frogs}

\author{
Kosuke Suda, Shun R. Hayashi, Kei Tamura, Nobuhiko Takamatsu and Michihiko Ito* \\ Department of Bioscience, School of Science, Kitasato University, Sagamihara, Japan
}

Interspecific hybridization between two closely related species sometimes resulted in a new species with allotetraploid genomes. Many clawed frog species belonging to the Xenopus genus have diverged from the allotetraploid ancestor created by the hybridization of two closely related species with the predicted $L$ and $S$ genomes. There are speciesspecific repeated sequences including transposable elements in each genome of organisms that reproduce sexually. To understand what happened on and after the hybridization of the two distinct systems consisting of repeated sequences and their corresponding piRNAs, we isolated small RNAs from ovaries and testes of three Xenopus species consisting of allotetraploid $X$. laevis and $X$. borealis and diploid $X$. tropicalis as controls. After a comprehensive sequencing and selection of piRNAs, comparison of their sequences showed that most piRNA sequences were different between the ovaries and testes in all three species. We compared piRNA and genome sequences and specified gene clusters for piRNA expression in each genome. The synteny and homology analyses showed many distinct piRNA clusters among the three species and even between the two $L$ and/or S subgenomes, indicating that most clusters of the two allotetraploid species changed after hybridization. Moreover, evolutionary analysis showed that DNA transposons including Kolobok superfamily might get activated just after hybridization and then gradually inactivated. These findings suggest that some DNA transposons and their piRNAs might greatly influence allotetraploid genome evolution after hybridization.

Keywords: hybrid, transposable elements, piRNA cluster, allotetraploidization, reproductive isolation

\section{INTRODUCTION}

Interspecific hybridization, which is the crossing of two species, plays an important role in evolution. Many events of whole genome duplication (WGD) occurred during interspecific hybridization. For example, hybridization was believed to be involved in the two-rounds of WGD in the common ancestor of vertebrates (Ohno, 1970; Holland et al., 1994; Spring, 1997). Recently, Simakov et al. (2020) reported that the second WGD occurred in the mid-late Ordovician by interspecific hybridization. The allotetraploid frog Xenopus laevis diverged from the hybrid between two closely related diploid Xenopus species. Session et al. (2016) reported that the allotetraploid $X$. laevis origin should have had two distinct subgenomes with distinct families of transposable elements (TEs), and that the two diploid progenitor species diverged about 34 million years ago (mya) and hybridized approximately 17-18 mya. Hybridogenesis, the elimination of one of the parental 
genomes in the germline cells and gamete production of the other parental species is another interesting phenomenon in interspecific hybridization as observed in the edible frog Pelophylax esculentus (Miura et al., 2021).

We speculated that there are some significant differences of repeated sequences including transposons between two closelyrelated species, although the two genomes should have only a few differences of gene sequences. Transposons, also known as transposable elements (TEs), are mobile DNA sequences that can proliferate and are scattered in the genomes. Most multicellular organisms possess a large proportion of TEs in their genomes. Their selfish activity induces genome instability in various cells, including germ line cells (Belancio et al., 2008). In particular, genomic information in germ line cells should be properly inherited for subsequent generations. It has been known that PIWI-interacting RNAs (piRNA) belong to small noncoding RNAs and function in protection of genomic information from transposons in animal germ line cells. The complexes between piRNAs and PIWI are involved in epigenetic and posttranscriptional silencing of transposons against their expansion and invasion (Klattenhoff et al., 2007; Seto et al., 2007; Siomi et al., 2011). Precursor piRNAs are processed into mature piRNAs consisting of 25-32 nucleotides. Precursor piRNAs are transcribed from genomic regions termed piRNA clusters, which contain various types of transposons (Siomi et al., 2011). Transposons vary and evolve dramatically across species. Thus, piRNA-encoding piRNA clusters must have evolved concomitantly. However, little is known about the molecular coevolution of piRNA clusters and transposons. Moreover, the influence of interspecific hybridization on the transposon-piRNA system in vertebrates has not been reported, yet.

How did the mixture of the two different transposon-piRNA systems by interspecific hybridization influenced Xenopus allotetraploid genome evolution? To clarify the evolutionary overview of transposons and transposon-derived piRNAs in genomes after interspecific hybridization, we first identified piRNAs by small RNA-seq from female and male gonads of three Xenopus species consisting of hybrid-derived allotetraploid species, $X$. laevis and $X$. borealis, and one diploid species $X$. tropicalis as a non-hybrid control. An ancestral species of $X$. tropicalis diverged from the common one between $X$. laevis and $X$. borealis about 48 mya (Session et al., 2016). From the piRNA sequence and genome information, we found that piRNA clusters were not well conserved between the $\mathrm{L}$ and $\mathrm{S}$ subgenomes of $X$. laevis. Moreover, evolutionary analysis of transposons indicated that some DNA transposon superfamilies might become active just after hybridization. Finally, we discuss the relationships between "transposon-piRNA" system and interspecific hybridization.

\section{MATERIAL AND METHODS}

\section{Databases}

Genomic sequence information from $X$. laevis and $X$. tropicalis was obtained from Xenbase (www.xenbase.org/entry/), including $X$. laevis v9.2 and X. tropicalis v9.1 genome assemblies. Repbase (www.girinst.org/repbase/) was used for the transposon libraries of $X$. laevis and $X$. tropicalis. The Xenopus transposon library was constructed by merging the two libraries.

\section{Small RNA Extraction and Small RNA-Seq}

Ovaries from adult female frogs were cut into small pieces, flashfrozen in liquid nitrogen, and stored. Testes from adult male frogs were flash-frozen in liquid nitrogen. Small RNAs were purified using a small RNA isolation kit (ISOGEN II, Nippon gene, Japan) according to the manufacturer's protocol, except for the percentage change in ethanol from 75 to $90 \%$. piRNAs were separated on a $15 \%$ polyacrylamide TBE-urea gel, and 20-50 base pair long were excised from the gel. They were then recovered using a small RNA PAGE recovery kit (Zymo Research, California, United States). Library construction and small RNA sequences were obtained from DNAFORM (Yokohama, Japan).

\section{Genome Assembly and Gene Annotation of $X$. borealis}

Scaffolds were assembled using ABySS (github.com/bcgsc/abyss) under the conditions of $(\mathrm{k}=83 \mathrm{~B}=30 \mathrm{G} \mathrm{H}=3 \mathrm{kc}=3 \mathrm{v}=-\mathrm{v}$ ) with $X$. borealis female or male whole genome sequences, SRR6357673 and SRR6357672, respectively. The resulting female and male scaffolds were integrated, and reference-guided scaffolding was performed with RaGOO (Alonge et al., 2019) -T sr using X. laevis v9.2 genome assembly as a reference. Gene annotation was carried out using BRAKER (Hoff et al., 2019). Protein sequence data were obtained from Xenopus laevis v2 (GCA_001663975.1_Xenopus_laevis_v2/), and the annotation data were filtered using gFACS (Caballero and Wegrzyn, 2019) at two statuses (monoexonic gene: min-exon-size 20 min-intronsize 20 min-CDS-size 900 -statistics-at-every-step-noprocessing-rem-multiexonics-rem-genes-without-start-andstop-codon-canonical-only, multiexonic gene: -min-exon-size $20 \mathrm{~min}$-intron-size $20 \mathrm{~min}$-CDS-size 300 -statistics-at-everystep-no-processing-rem-monoexonics-rem-genes-withoutstart-and-stop-codon-canonical-only). Gene protein sequences were extracted using SeqKit (bioinf.shenwei.me/seqkit/). Predicted protein sequences were aligned to those in $X$. laevis using BLASTP and then filtered using an E-value $<0.05$.

\section{Classification of DNA Sequences in Genomes}

In each genome, repeated sequences were mapped and classified using RepeatMasker as described above, and then exons, introns, and others were specified with BEDTools intersect.

\section{Identification of Transposable Elements in Genomes}

The genomic transposon library from $X$. borealis was constructed using RepeatModeler (github.com/Dfam-consortium/TETools). The resulting $X$. borealis transposon library was incorporated into the transposon library, which was described above. The genomic locations of transposons were identified using RepeatMasker (www.repeatmasker.org/). Transposon diversity was 
recalculated using MAFFT (www.mafft.cbrc.jp/alignment/ software/) -retree 2 -reorder and FastTree (www. microbesonline.org/fasttree/) -nt. A customized Python script was used for overlapping locations of transposons to give priority to low diversity scores. TE landscapes were basically generated by using RepeatLandscape (github.com/caballero/ RepeatLandscape) to calculate Kimura distance as a measure of age.

\section{Identification of piRNAs and Their Clusters}

Protein-coding RNAs and miRNAs were excluded from small RNA raw sequence reads using unitas that is out-of-the-box ready software for complete annotation of small RNA sequence datasets (Gebert et al., 2017), and 24 to 32 base reads were extracted using SeqKit (bioinf.shenwei.me/seqkit/). The resulting RNA reads were mapped to genome sequence data using Bowtie (bowtiebio.sourceforge.net/index.shtm) -1.18 -n 0 -a-best-strata, and then treated with customized Python script, which allows alignments with two mismatches in the area except for the seed sequence and the last two sequences under the condition of sorted ELAND format in exchange of SAM format. In each read, one minimum mismatch alignment was selected with customized Python script. The counts of the resulting selected alignments mapped on the specific chromosome position were recalculated using reallocate (www.smallrnagroup.uni-mainz.de/ software.html) $100001000 \mathrm{~b}$ 1. Candidates of piRNA clusters were selected by using proTRAC (Rosenkranz and Zischler, 2012). The clusters were then identified by removing piRNAs that were lower than 1 loci/kb and 10 loci/piRNA clusters with awk and customized python scripts. Because proTRAC was useful for detecting a high density of piRNAs in a relatively wide region of the genome, we also used Stringtie (Pertea et al., 2015) for detecting the clusters in a relatively narrow region of the genome as performed by Toombs et al. (2017). In X. tropicalis and X. laevis, they were identified using customized Python script under the conditions of (Stringtie -m 50 -g 50 and -merge -m 500 -g 250 -F 50). In $X$. borealis, whose genome information has many $100 \mathrm{bp}$ gaps, piRNA clusters were detected using customized Python script under the conditions of (Stringtie -m 150 -g 50 and -merge -m 500 -g 250 -F 50). In summary, piRNA clusters were determined by detection using proTRAC and/or Stringtie. piRNA clusters with more than half of the overlapped sequences in the specified region between female and male gonads were defined as the common ones.

\section{Analysis of $1 \mathrm{U} / 10 \mathrm{~A}$ and Overlapped Plus/ Minus piRNA Strands}

10 base DNA sequence corresponding to $5^{\prime}$ of each piRNA was extracted in the recalculated mapping ELAND format file which was described in the above paragraph, and changed to RNA sequence using seqkit seq-dna2ma. $1 \mathrm{U} / 10 \mathrm{~A}$ was plotted using WebLogo (weblogo.berkeley.edu).

For plus/minus strands of piRNAs, the ELAND format file was exchanged into BED format. Then, using awk script, each piRNA sequence was mapped in the genome and identified as plus or minus strand. Overlapped piRNA sequences were found using
BEDTools intersect (Quinlan and Hall. 2010). The overlap score was measured as the product of plus and minus reads. The $10 \mathrm{nt}$ Z-score was calculated from the mean and standard deviation of ratio of the overlap score on each length to total ones.

\section{Component of Genome and piRNA Clusters}

Gene and exon loci were extracted for each Xenopus gene annotation and overlapped loci were merged using BEDTools merge. The classification of the genome and piRNA clusters were done in order of transposon, gene, exon, to avoid overlapping other components with BEDTools intersect.

\section{Evolutionary Analysis of piRNA Clusters}

Synteny analysis of piRNA clusters with lengths longer than $1 \mathrm{~kb}$ was performed as follows. First, localizations of 10 exons up- and downstream of piRNA clusters were examined in the corresponding genome, and the genomic sequences were extracted. Neighboring BLAST hits were grouped into contiguous exons. Second, syntenic relationships between piRNA clusters were verified using more than three homologous exons and their bit scores. Third, we identified homologous sequences to piRNA clusters and their corresponding transposons in the query genome using blastntask dc-megablast. BLAST hits below the relative size (10\%) were discarded.

\section{RESULTS}

\section{A Variety of piRNA Molecules From Ovaries and Testes in the Three Xenopus Species, $X$. tropicalis, $X$. leavis, and $X$. borealis}

Predicted piRNA molecules were selected from comprehensive sequences of small RNAs from the ovaries and testes of the three Xenopus species (Section 2; Figure 1). Almost all the sequences have two characteristics of piRNAs, a high relative amount of $10 \mathrm{nt}$ overlap from $5^{\prime}$ ends and 1 U/10 A biases (Figure 2). Most of the piRNA sequences from each gonad of the three species contained similar numbers of plus and minus strand sequences at every nucleotide length (Supplementary Figure S1), suggesting that they were produced through ping-pong cycles. We also found that there were more than $10^{7}$ piRNAs in each gonad from the three species, and more than $90 \%$ of the piRNAs were distinct between the ovary and testis from each species (Supplementary Figure S2).

\section{Most piRNA Sequences Are Derived From Retrotransposons, but Also DNA Transposons and Genes in the Xenopus Gonads}

Most piRNAs are believed to be derived from TEs. We then examined the origin of each piRNA sequence in $X$. tropicalis, $X$. laevis, and $X$. borealis. We first classified the three genomic DNAs into eight groups: DNA transposons, retrotransposons, satellite DNA, simple repeat DNA, exons, introns, others, and unknown 


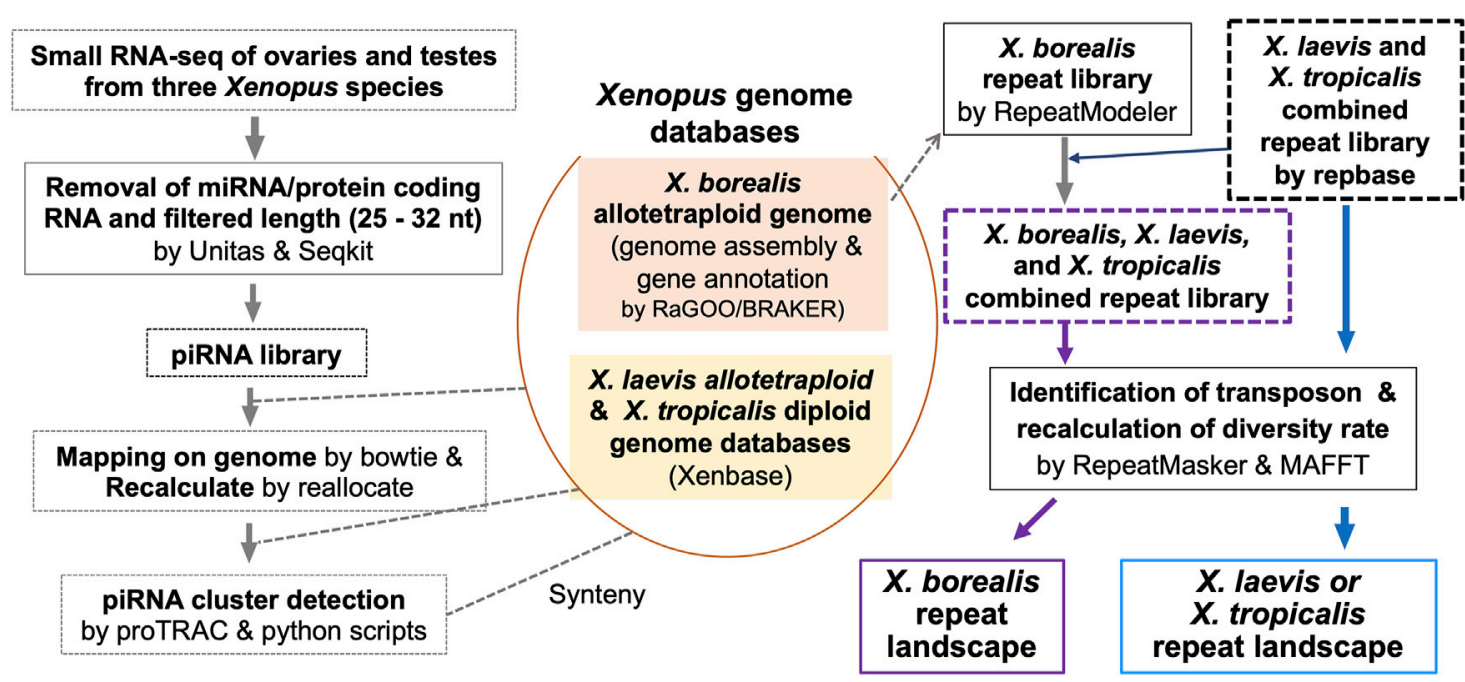

FIGURE 1 | Strategy for genome-wide analysis of piRNAs and repeat sequences including TEs in a diploid and two allotetraploid Xenopus frog species.

sequences in each genome (Section 2). We found that there were similar composition percentages in selfish DNA in all three Xenopus genomes: $23-26 \%$ of DNA transposons, $8.3-8.8 \%$ of retrotransposons, and less than $2 \%$ of satellite DNA or simple repeats (Table 1). The percentage of exons (1.9\%) and introns (10\%) in $X$. borealis were much lower than those $(5.0-5.6 \%$ and $28-30 \%$, respectively) of the other two species, maybe because "others" in X. borealis occupied a higher percentage (50\%) from the insufficiency of genome assembly data (Table 1).
Next, we mapped the obtained piRNA sequences to the classified genomes. We found that all of the DNA transposon-, retrotransposon-, exon-, and intron-derived piRNAs from the ovaries and testes of the three species shared the two characteristics of piRNAs as the unclassified piRNAs did in Figure 2 (Supplementary Figure S3). Interestingly, retrotransposon-derived piRNAs occupied higher percentages in the ovaries (37-41\%) than in the testes $(20-25 \%)$ in total piRNAs in all three species (Table 1). In

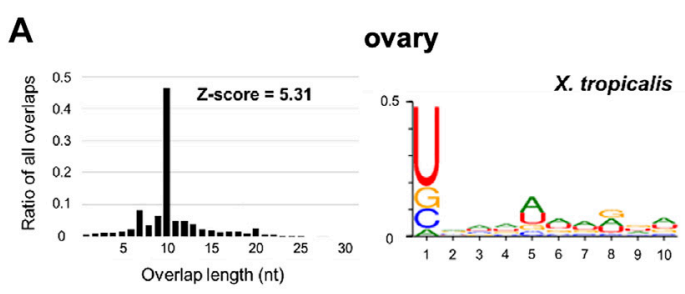

B
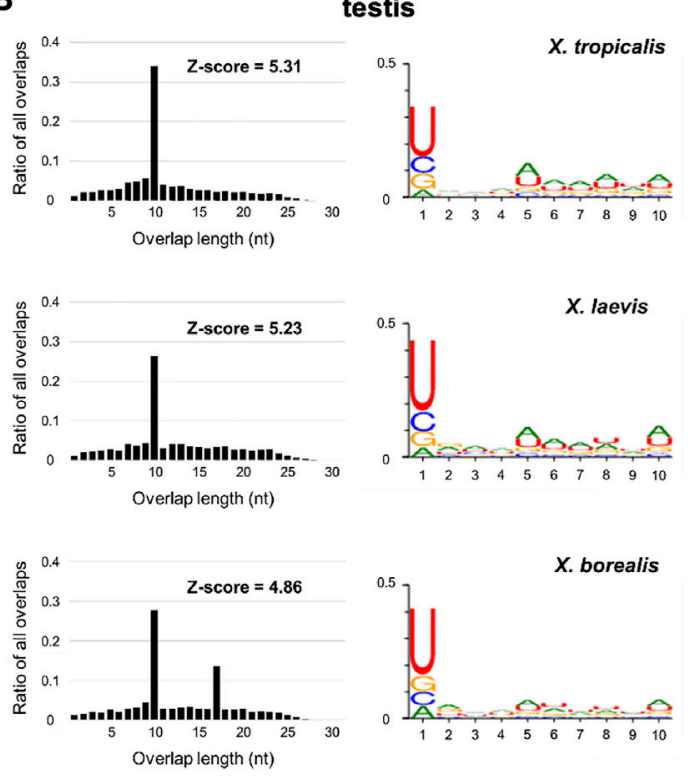

testis

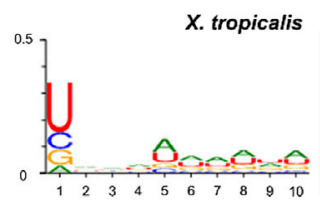

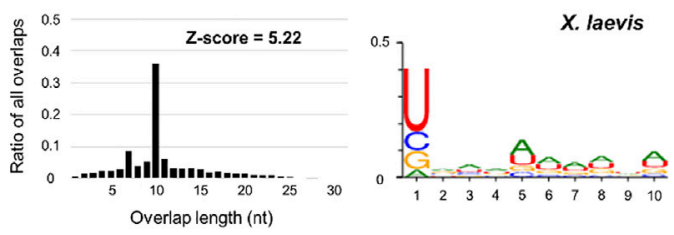
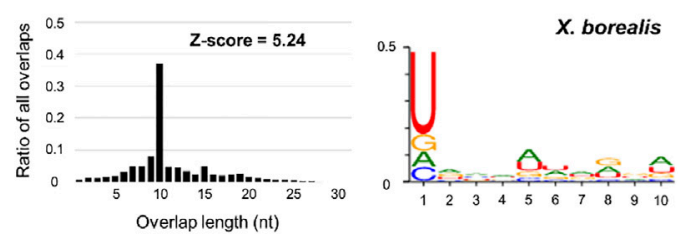

FIGURE 2 | Sequence characterization of ovarian and testicular piRNAs in the three Xenopus species, $X$. tropicalis, $X$. laevis, and $X$. borealis. Distributions of $5^{\prime}$ overlap lengths (left) and $1 \mathrm{U} / 10 \mathrm{~A}$ bias (right) on piRNA molecules transcribed in ovaries (A) and testes (B). The amounts of piRNAs were normalized by $10^{7} /$ mapped $^{2}$ piRNA counts in length distribution. Numbers of sequence types of piRNAs transcribed in testes and ovaries are shown in Venn diagrams (C). 
TABLE 1 | Proportion (\%) of each DNA or RNA component in genomic DNA or total piRNAs of $X$. tropicalis $(X t)$, $X$. laevis $(X)$, and $X$. borealis $(X b)$.

\begin{tabular}{|c|c|c|c|c|c|c|c|c|c|}
\hline & \multicolumn{3}{|c|}{$\%$ of genome } & \multicolumn{3}{|c|}{ Ovary ( $\%$ of total piRNAs) } & \multicolumn{3}{|c|}{ Testis ( $\%$ of total piRNAs) } \\
\hline & $\mathbf{X t}$ & $\mathbf{X I}$ & $\mathbf{X b}$ & $\mathbf{X t}$ & $\mathbf{X I}$ & $\mathbf{X b}$ & $\mathbf{X t}$ & $\mathbf{X I}$ & $\mathbf{X b}$ \\
\hline DNA transposon & 24 & 26 & 23 & 18 & 8.7 & 8.9 & 8.5 & 10 & 11 \\
\hline Retro transposon & 8.8 & 8.3 & 8.6 & 40 & 37 & 41 & 23 & 20 & 25 \\
\hline Satellite & 1.7 & 0.40 & 1.8 & 0.027 & 0.039 & 4.4 & 0.094 & 0.011 & 0.34 \\
\hline Simple repeat & 0.86 & 0.68 & 1.2 & 0.27 & 0.41 & 0.76 & 0.39 & 0.66 & 0.79 \\
\hline Unknown & 0.33 & 0.22 & 4.0 & 0.43 & 0.083 & 3.4 & 0.16 & 0.089 & 1.8 \\
\hline Exon & 5.6 & 5.0 & 1.9 & 6.6 & 3.4 & 4.3 & 14 & 8.9 & 7.6 \\
\hline Intron & 30 & 28 & 10 & 21 & 18.4 & 12 & 27 & 28 & 16 \\
\hline Other & 29 & 32 & 50 & 14 & 32 & 25 & 27 & 32 & 37 \\
\hline
\end{tabular}

addition, we found that DNA transposon- and gene (intron and exon)-derived piRNAs were substantially transcribed in both the ovaries and testes of the three Xenopus species (Table 1), although piRNAs have been reported to be mostly transcribed from retrotransposons within mammalian genomes (Girard et al., 2006).

\section{piRNA Clusters Are Not Well Conserved Between the Allotetraploid $L$ and $S$ Subgenomes}

Most piRNAs are believed to be transcribed from genomic piRNA clusters (Rosenkranz et al., 2021). We then identified piRNA clusters in the three Xenopus genomes from the ovarian and testicular piRNA sequences (Section 2; Figure 1). The number of piRNA clusters was approximately 14,17 , and 13 thousand in the diploid $X$. tropicalis, allotetraploid $X$. laevis, and $X$. borealis, respectively (Supplementary Table S1), indicating larger numbers in $X$. tropicalis in view of genome size. Interestingly, in all three species, the relatively higher density regions of the piRNA clusters were distinct between each ovary and testis, and more than half of the piRNA clusters transcribed specific expression in the ovaries or testes (Supplementary Figure S4; Supplementary Tables S1).

The piRNA clusters are conserved across related species in mammals (Girard et al., 2006; Gebert et al., 2019). To clarify the conservation of piRNA clusters during Xenopus diversification, we performed synteny and homology analyses of these clusters not only among the three Xenopus species but also between the $\mathrm{L}$ and $S$ subgenomes of the two allotetraploid species. In view of chromosomal locations, the piRNA clusters were moderately conserved (approximately 33-59\%) in every combination (Supplementary Table S2).

We next examined each proportion of the eight types of DNA, as shown in Table 1, in the ovarian or testicular piRNA clusters from the three Xenopus species (Supplementary Figure S5). The proportion of piRNA DNA to retrotransposons in the piRNA clusters was more than two times higher than that of retrotransposon DNA in the genomes of the three species. In contrast, the proportion of piRNAs to DNA transposons in the piRNA clusters was slightly lower than that of DNA transposonderived DNA in all (sub)genomes. In addition, there were few significant differences in their compositions between the L and S subgenomes of the two allotetraploid species.

\section{Distinct Landscapes of DNA Transposons and Retrotransposons Between the Diploid and Allotetraploid Xenopus Species}

The findings of the piRNAs and their clusters in the three Xenopus species (Supplementary Figures S2, S4; Supplementary Tables S1, S2) indicate their divergent evolution, suggesting drastic evolution of "transposon-piRNA" system during Xenopus species diversity. To clarify the fast and drastic evolution of TEs during species diversification in the Xenopus frogs, we examined their nucleotide variations using the genome information of the three species and constructed a TE landscape from their variation frequencies in each genome (Section 2; Figure 1). Most inactive TEs are selectively neutral. Therefore, we adopted 0.01 nucleotide substitution to 3.23-3.33 mya as molecular clock, which was reported in Xenopus species by Session et al. (2016). Accordingly, 17 and 34 mya in Figure 3 correspond to the times during the hybridization between the two diploid species with the L- an S-derived genomes and the speciation time to the two ancestral species, respectively. The landscapes of TEs in the X. tropicalis diploid genome and L/S subgenomes from allotetraploid $X$. laevis and $X$. borealis are shown in Figure 3. The landscape of the $X$. tropicalis genome was completely different from that of the allotetraploid L/S subgenomes. Both the DNA transposons and retrotransposons appeared to be active until recently in $X$. tropicalis (Figure 3A). In contrast, $X$. laevis and $X$. borealis $\mathrm{L}$ and $S$ subgenomes have similar landscapes (Figures 3B,C). Interestingly, the four subgenomes shared large peaks of DNA transposons around 17 mya. These findings indicate that the mixture of the two genomes might be involved in fast and drastic evolution of TEs, especially DNA transposons.

\section{Activation of a DNA Transposon Superfamily Kolobok Around the Interspecific Hybridization}

Since DNA transposons appeared to be activated around the hybridization in the common ancestor of $X$. laevis and $X$. 

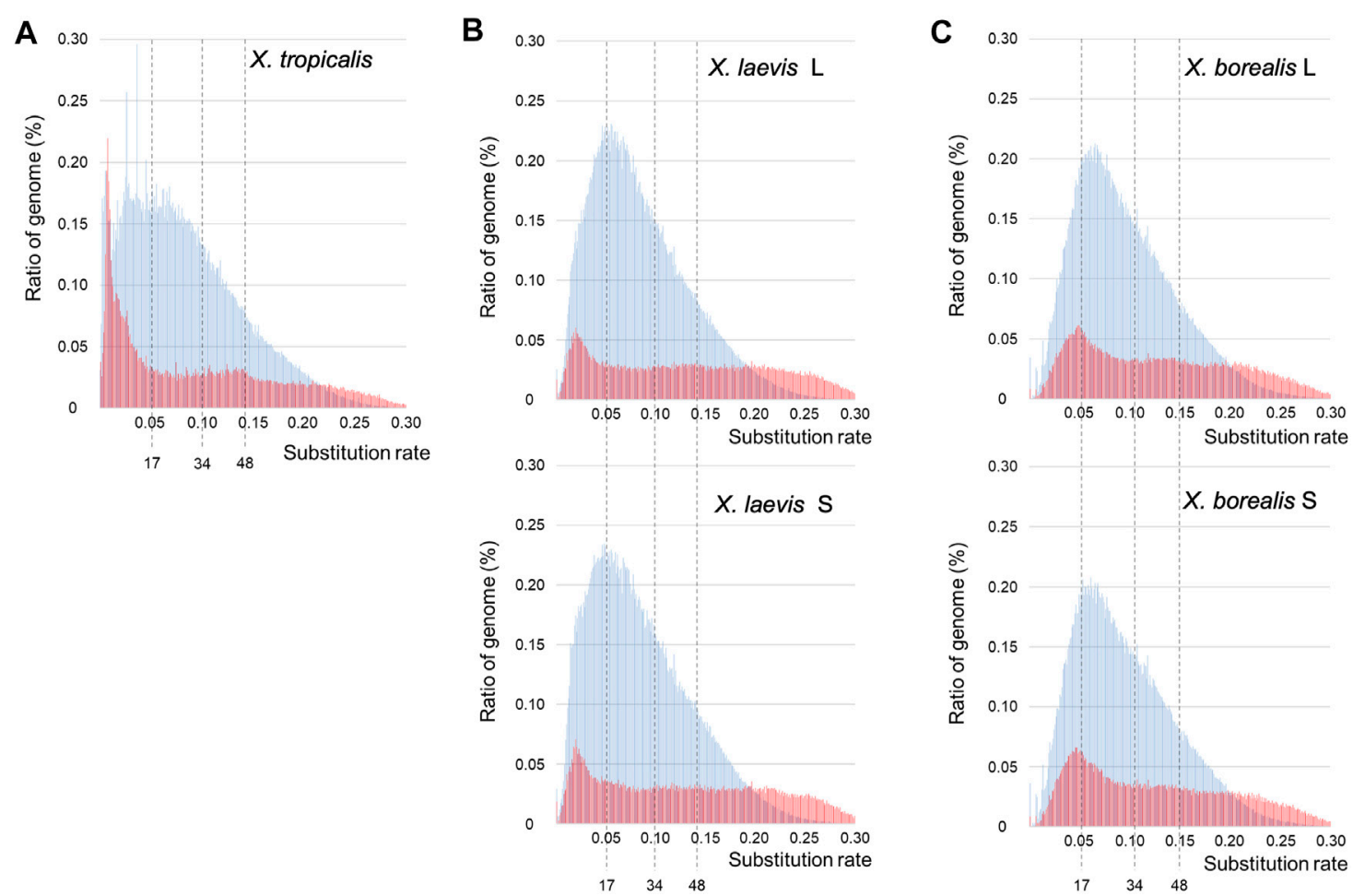

FIGURE 3 | Landscapes of DNA transposons and retrotransposons in the three Xenopus species. The substitution ratios of DNA transposons (blue) and retrotransposons (red) in X. tropicalis (A), X. laevis $L$ and $S \mathbf{~ ( B ) , ~ a n d ~ X . ~ b o r e a l i s ~} L$ and $S(\mathbf{C})$ are shown on the horizontal axis. Proportion (\%) of the transposons to each genome DNA are shown on the vertical axis. The numbers 34 and 17 indicate speciation and hybridization time (mya) of the predicted L and S species, which correspond to the substitution ratios, 0.10 and 0.05 , respectively.
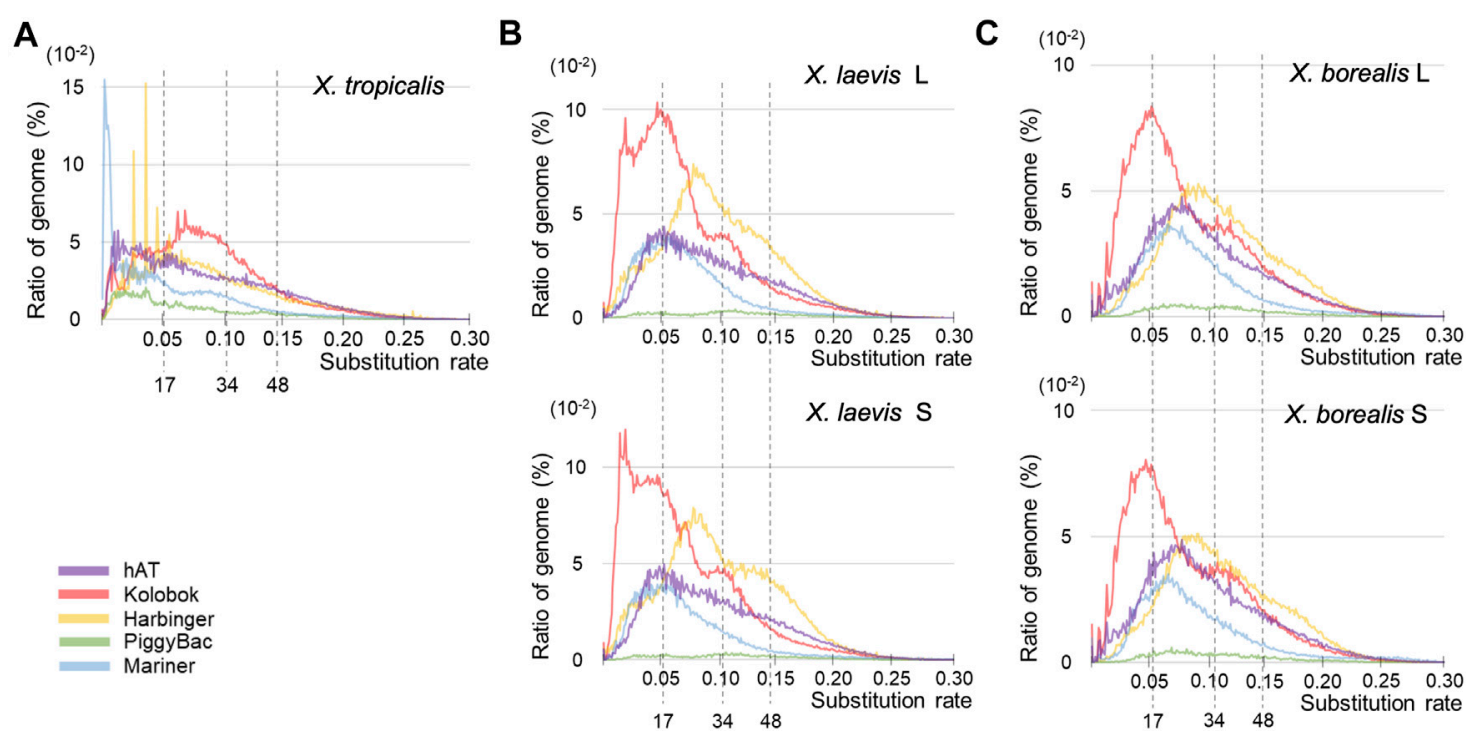

FIGURE 4 | Landscapes of six major superfamilies of DNA transposons in the three Xenopus species. The substitution ratio of each superfamily in $X$. tropicalis (A),

$X$. laevis $L$ and $S(\mathbf{B})$, and $X$. borealis $L$ and $S(\mathbf{C})$ are shown on the horizontal axis. Proportion (\%) of the transposons to each genome DNA are shown on the vertical axis. 
borealis followed by inactivation, we analyzed sequence variations of five major superfamilies (Figure 4). Similar to the results in Figure 3, the landscape from $X$. tropicalis was unique (Figure 4A), whereas those of the five superfamilies were similar between the two $\mathrm{L}$ and $\mathrm{S}$ subgenomes of $X$. laevis and $X$. borealis (Figures 4B,C). Notably, the Kolobok superfamily showed activity peaks at approximately 17 mya in both $X$. laevis L/S and $X$. boralis L/S subgenomes. The Kolobok superfamily also showed another peak around 7 mya in the $\mathrm{L}$ and $\mathrm{S}$ subgenomes in $X$. laevis, but not in $X$. borealis. In contrast, activity peaks around 17 mya were observed in $h A T$ and Mariner superfamilies from $X$. laevis L/S, but not in $X$. borealis $\mathrm{L} / \mathrm{S}$ subgenomes. These findings suggest that the high activity of some transposon superfamilies including Kolobok could be involved in the hybridization event with the mingled state of two distinct genomes.

\section{DISCUSSION}

In this study, we obtained ovarian and testicular piRNA sequences from the three Xenopus species and analyzed them (Figure 2; Supplementary Figures S1-S5; Supplementary Tables S1, S2). All of the samples have two characteristics of piRNAs, $1 \mathrm{U} / 10 \mathrm{~A}$ bias and the peak of 10 nucleotide overlap from $5^{\prime}$ (Figure 2). Curiously, we observed not only 10, but also 17 significant overlaps in the $X$. borealis testicular piRNAs (Figure 2). However, we could not understand the reason. Less than $10 \%$ of the total types of piRNAs were common between the ovary and testis in each species (Supplementary Figure S2). We do not know whether the differences between them was caused mainly by female and male gonads or each individual. In several actinopterygian fish, ovary and testis-specific piRNAs have been reported (Wang et al., 2016; Yuan et al., 2019). Faunes et al. (2012) reported that in X. tropicalis a large number of transposon-derived piRNAs are present in gastrula embryos, most of which are shared with oocytes. Furthermore, satellite repeat-derived piRNAs regulate the embryonic development of an insect (Halbach et al., 2020). It will be interesting to study whether some oocyte-derived piRNAs control embryogenesis, such as repression of selfish DNA and RNA.

By mapping the piRNA sequences to their corresponding genomes, we specified the piRNA clusters transcribed in female and male gonads in the three Xenopus species, indicating that almost all the piRNA clusters were not well conserved among the three species or between the allotetraploid-derived $\mathrm{L}$ and $\mathrm{S}$ subgenomes (Table 1; Supplementary Tables S1, S2; Supplementary Figure S4). Therefore, piRNA clusters might have evolved drastically during species diversity in Xenopus frogs. Toombs et al. (2017) reported that Xenopus piRNAs were identified as PIWI-interacting RNAs from early stage oocytes and that PIWI could promote the turnover of TEs and other RNAs and regulate mRNA localization and translation in germ cells. Because the positions of the piRNA clusters were not shown in the report, we could not directly compare their data with those obtained in this study. Then we compared the piRNA clusters between the updated database of piRNA cluster (www. smallrnagroup.uni-mainz.de/piRNAclusterDB/) and this study. More than half of piRNA clusters (391/566 and 302/ $480)$ in the updated database from $X$. laevis and X. tropicalis, respectively, were merged to those identified in this study. The differences might be supported in part by the fact that the frogs in both the species were not derived from the inbred lines. Gebert et al. (2019) reported that some piRNA clusters were well conserved during primate evolution. This difference might be related to the higher percentage of DNA transposons in Xenopus frog genomes, as shown in Table 1, than in primate genomes.

Although piRNAs and PIWI are well known to silence retrotransposons by heterochromatin formation of their genes or cleavage of their transcripts (Russell and LaMarre, 2021), it remains unclear whether some piRNA molecules could regulate DNA transposons and how they would do so. In many mammals, the majority of TEs originate from retrotransposons (Vandewege et al., 2016). In contrast, DNA transposons are dominant among TEs in amphibian genomes (Hellsten et al., 2010; Session et al., 2016; Table 1). Here, we found that DNA transposon-derived piRNAs accounted for $5-12 \%$ of total piRNAs in female and male gonads from the three Xenopus species (Table 1). It is interesting to study whether DNA transposon-derived piRNAs could regulate DNA transposons during gametogenesis and embryogenesis and how they would do so.

Interspecific hybridization has been reported to cause the proliferation of transposons in some bilaterian animal hybrids such as fruit flies in the Drosophila genus and teleost fish sculpins in the Cottus genus (Khurana et al., 2011; Dennenmoser et al., 2019; Kotov et al., 2019). In the TE landscapes, large peaks of total DNA transposons were observed around the hybridization time (17 mya) in the two hybrid-derived species $X$. laevis and $X$. borealis, but not in the diploid species $X$. tropicalis (Figure 3). Moreover, we observed that several DNA transposon superfamilies including Kolobok were activated around the hybridization time (Figure 4). In general, the genomes of two closely related species had few differences in gene sequences, but contain distinct TEs and piRNA genes, including piRNA clusters. Here, we confirmed the differences in the piRNA clusters in the three Xenopus genomes (Supplementary Table S2). The interspecific hybridization, which was accompanied with the mixture of the two distinct Xenopus genomes, might induce activation of DNA transposons such as the Kolobok superfamily because of the two distinct "transposon-piRNA" systems. The activities of the two superfamilies were repressed by their corresponding piRNAs that newly emerged piRNA genes should encode. We speculate that the asymmetric evolution between the $\mathrm{L}$ and $\mathrm{S}$ subgenomes in the allotetraploid Xenopus species has been caused, in part, by activation of the DNA transposon and repression of their corresponding piRNAs around and after hybridization. 


\section{DATA AVAILABILITY STATEMENT}

The datasets presented in this study can be found in online repositories. The names of the repository/repositories and accession number(s) can be found below: DNA data bank (DDBJ), PRJDB12462.

\section{ETHICS STATEMENT}

The animal study was reviewed and approved by The Institutional Animal Care and Use Committee of Kitasato University. Written informed consent was obtained from the owners for the participation of their animals in this study.

\section{AUTHOR CONTRIBUTIONS}

KS and MI designed the study. KS, SH, KT, NT, and MI analyzed the data. KS and MI wrote the paper.

\section{REFERENCES}

Alonge, M., Soyk, S., Ramakrishnan, S., Wang, X., Goodwin, S., Sedlazeck, F. J., et al. (2019). RaGOO: Fast and Accurate Reference-Guided Scaffolding of Draft Genomes. Genome Biol. 20, 224. doi:10.1186/s13059-019-1829-6

Belancio, V. P., Hedges, D. J., and Deininger, P. (2008). Mammalian Non-LTR Retrotransposons: for Better or Worse, in Sickness and in Health. Genome Res. 18, 343-358. doi:10.1101/gr.5558208

Caballero, M., and Wegrzyn, J. (2019). gFACs: Gene Filtering, Analysis, and Conversion to Unify Genome Annotations across Alignment and Gene Prediction Frameworks. Genomics, Proteomics \& Bioinformatics 17, 305-310. doi:10.1016/j.gpb.2019.04.002

Dennenmoser, S., Sedlazeck, F. J., Schatz, M. C., Altmüller, J., Zytnicki, M., and Nolte, A. W. (2019). Genome-wide Patterns of Transposon Proliferation in an Evolutionary Young Hybrid Fish. Mol. Ecol. 28, 1491-1505. doi:10.1111/mec. 14969

Faunes, F., Almonacid, L. I., Melo, F., and Larrain, J. (2012). Characterization of Small RNAs inXenopus Tropicalisgastrulae. Genesis 50, 260-270. doi:10.1002/ dvg.22012

Gebert, D., Hewel, C., and Rosenkranz, D. (2017). Unitas: the Universal Tool for Annotation of Small RNAs. BMC Genomics 18, 644. doi:10.1186/s12864-0174031-9

Gebert, D., Zischler, H., and Rosenkranz, D. (2019). Primate piRNA Cluster Evolution Suggests Limited Relevance of Pseudogenes in piRNA-Mediated Gene Regulation. Genome Biol. Evol. 11, 1088-1104. doi:10.1093/gbe/ evz060

Girard, A., Sachidanandam, R., Hannon, G. J., and Carmell, M. A. (2006). A Germline-specific Class of Small RNAs Binds Mammalian Piwi Proteins. Nature 442, 199-202. doi:10.1038/nature04917

Halbach, R., Miesen, P., Joosten, J., Taşköprü, E., Rondeel, I., Pennings, B., et al. (2020). A Satellite Repeat-Derived piRNA Controls Embryonic Development of Aedes. Nature 580, 274-277. doi:10.1038/s41586-020-2159-2

Hellsten, U., Harland, R. M., Gilchrist, M. J., Hendrix, D., Jurka, J., Kapitonov, V., et al. (2010). The Genome of the Western Clawed Frog Xenopus Tropicalis. Science 328, 633-636. doi:10.1126/science.1183670

Hoff, K. J., Lomsadze, A., Borodovsky, M., and Stanke, M. (2019). Whole-Genome Annotation with BRAKER. Methods Mol. Biol. 1962, 65-95. doi:10.1007/978-14939-9173-0_5

Holland, P. W. H., Garcia-Fernàndez, J., Williams, N. A., and Sidow, A. (1994). Gene Duplications and the Origins of Vertebrate Development. Dev. Suppl. 1994, 125-133. doi:10.1242/dev.1994.supplement.125

\section{FUNDING}

This work was partially supported by Grant-in-Aid for Scientific Research, Japan Society for the Promotion of Science (No. 18K06389) to MI and Takahashi Industrial and Economic Research Foundation, Japan to MI.

\section{ACKNOWLEDGMENTS}

We would like to thank Hiroshima University Amphibian Research Center for providing $X$. tropicalis frogs.

\section{SUPPLEMENTARY MATERIAL}

The Supplementary Material for this article can be found online at: https://www.frontiersin.org/articles/10.3389/fgene.2022.766424/ full\#supplementary-material

Khurana, J. S., Wang, J., Xu, J., Koppetsch, B. S., Thomson, T. C., Nowosielska, A., et al. (2011). Adaptation to P Element Transposon Invasion in Drosophila melanogaster. Cell 147, 1551-1563. doi:10.1016/j.cell.2011.11.042

Klattenhoff, C., Bratu, D. P., McGinnis-Schultz, N., Koppetsch, B. S., Cook, H. A., and Theurkauf, W. E. (2007). Drosophila rasiRNA Pathway Mutations Disrupt Embryonic axis Specification through Activation of an ATR/Chk2 DNA Damage Response. Dev. Cel 12, 45-55. doi:10.1016/j.devcel.2006.12.001

Kotov, A. A., Adashev, V. E., Godneeva, B. K., Ninova, M., Shatskikh, A. S., Bazylev, S. S., et al. (2019). piRNA Silencing Contributes to Interspecies Hybrid Sterility and Reproductive Isolation in Drosophila melanogaster. Nucleic Acids Res. 47, 4255-4271. doi:10.1093/nar/gkz130

Miura, I., Vershinin, V., Vershinina, S., Lebedinskii, A., Trofimov, A., Sitnikov, I. et al. (2021). Hybridogenesis in the Water Frogs from Western Russian Territory: Intrapopulation Variation in Genome Elimination. Genes 12, 244. doi:10.3390/genes12020244

Ohno, S. (1970). Evolution by Gene Duplication. Berlin: Springer.

Pertea, M., Pertea, G. M., Antonescu, C. M., Chang, T.-C., Mendell, J. T., and Salzberg, S. L. (2015). StringTie Enables Improved Reconstruction of a Transcriptome from RNA-Seq Reads. Nat. Biotechnol. 33, 290-295. doi:10.1038/nbt.3122

Quinlan, A. R., and Hall, I. M. (2010). BEDTools: a Flexible Suite of Utilities for Comparing Genomic Features. Bioinformatics 26, 841-842. doi:10.1093/ bioinformatics/btq033

Rosenkranz, D., Zischler, H., and Gebert, D. (2021). piRNAclusterDB 2.0: Update and Expansion of the piRNA Cluster Database. Nucleic Acids Res. 50, D259-D264. doi:10.1093/nar/gkab622

Rosenkranz, D., and Zischler, H. (2012). proTRAC - a Software for Probabilistic piRNA Cluster Detection, Visualization and Analysis. BMC Bioinformatics 13, 5. doi:10.1186/1471-2105-13-5

Russell, S. J., and LaMarre, J. (2018). Transposons and the PIWI Pathway - Genome Defence in Gametes and Embryos. Reproduction 156, R111-R124. doi:10.1530/ REP-18-0218

Session, A. M., Uno, Y., Kwon, T., Chapman, J. A., Toyoda, A., Takahashi, S., et al. (2016). Genome Evolution in the Allotetraploid Frog Xenopus laevis. Nature 538, 336-343. doi:10.1038/nature19840

Seto, A. G., Kingston, R. E., and Lau, N. C. (2007). The Coming of Age for Piwi Proteins. Mol. Cel 26, 603-609. doi:10.1016/j.molcel.2007.05.021

Simakov, O., Marlétaz, F., Yue, J.-X., O’Connell, B., Jenkins, J., Brandt, A., et al. (2020). Deeply Conserved Synteny Resolves Early Events in Vertebrate Evolution. Nat. Ecol. Evol. 4, 820-830. doi:10.1038/s41559-020-1156-Z

Siomi, M. C., Sato, K., Pezic, D., and Aravin, A. A. (2011). PIWI-interacting Small RNAs: the Vanguard of Genome Defence. Nat. Rev. Mol. Cel Biol. 12, 246-258. doi:10.1038/nrm3089 
Spring, J. (1997). Vertebrate Evolution by Interspecific Hybridisation - Are We Polyploid? FEBS Lett. 400, 2-8. doi:10.1016/s0014-5793(96)01351-8

Toombs, J. A., Sytnikova, Y. A., Chirn, G.-w., Ang, I., Lau, N. C., and Blower, M. D. (2017). Xenopus Piwi Proteins Interact with a Broad Proportion of the Oocyte Transcriptome. RNA 23, 504-520. doi:10.1261/rna.058859.116

Vandewege, M. W., Platt, R. N., Ray, D. A., and Hoffmann, F. G. (2016). Transposable Element Targeting by piRNAs in Laurasiatherians with Distinct Transposable Element Histories. Genome Biol. Evol. 8, 1327-1337. doi:10.1093/gbe/evw078

Wang, C. L., Wang, Z. P., Wang, J. Q., Li, M. Y., and Chen, X. W. (2016). Identification of Candidate piRNAs in the Gonads of Paralichthys olivaceus (Japanese Flounder). Zool Res. 37, 301-306. doi:10.13918/j.issn.2095-8137. 2016.5.301

Yuan, L., Li, L., Zhang, X., Jiang, H., and Chen, J. (2019). Identification and Differential Expression of piRNAs in the Gonads of Amur sturgeon (Acipenser Schrenckii). PeerJ 7, e6709. doi:10.7717/peerj.6709
Conflict of Interest: The authors declare that the research was conducted in the absence of any commercial or financial relationships that could be construed as a potential conflict of interest.

Publisher's Note: All claims expressed in this article are solely those of the authors and do not necessarily represent those of their affiliated organizations, or those of the publisher, the editors and the reviewers. Any product that may be evaluated in this article, or claim that may be made by its manufacturer, is not guaranteed or endorsed by the publisher.

Copyright (C) 2022 Suda, Hayashi, Tamura, Takamatsu and Ito. This is an open-access article distributed under the terms of the Creative Commons Attribution License (CC BY). The use, distribution or reproduction in other forums is permitted, provided the original author(s) and the copyright owner(s) are credited and that the original publication in this journal is cited, in accordance with accepted academic practice. No use, distribution or reproduction is permitted which does not comply with these terms. 